Pacific Journal of Mathematics

MINIMAL SPLITTING FIELDS FOR GROUP
REPRESENTATIONS. II 


\title{
MINIMAL SPLITTING FIELDS FOR GROUP REPRESENTATIONS, II
}

\author{
BURTON FEIN
}

\begin{abstract}
Let $p$ be an arbitrary prime and $m$ an arbitrary positive integer. A finite group $G$ is constructed which has an irreducible complex representation $T$ with character $\chi$ such that the Schur index of $\chi$ over $Q$ is $p$ but the minimum of [K: $Q(\chi)]$, taken over all abelian extensions $K$ of $Q$ in which $T$ is realizable, is $p^{m}$.
\end{abstract}

Let $Q$ denote the rationals and, for $n$ a pasitive integer, let $\varepsilon_{n}$ denote a primitive $n$th root of unity over $Q$. Let $\chi$ be the character afforded by a complex irreducible representation $T$ of a finite group $G$ of order $n$ and let $m_{Q}(\chi)$ denote the Schur index of $\chi$ over $Q$. In view of the famous theorem of $R$. Brauer that $T$ is realizable in $Q\left(\varepsilon_{n}\right)$, it is natural to ask how close to $m_{Q}(\chi)$ is $\min [L: Q(\chi)]$, where the minimum is taken over all subfields $L$ of cyclotomic extensions of $Q$ in which $T$ is realizable. Our main result shows that the above minimum is not, in general, very close to $m_{Q}(\chi)$.

THEOREM 1. Let $p$ be an arbitrary prime and $m$ an arbitrary positive integer. Then there exists a finite group $G$ of exponent $n$ and an irreducible complex representation $T$ of $G$ affording the character $\chi$ such that $m_{Q}(\chi)=p$ and $p^{m}=\min [L: Q(\chi)]$ where the minimum is taken over all abelian extensions $L$ of $Q$ in which $T$ is realizable. The minimum is attained at a subfield of $Q\left(\varepsilon_{n}\right)$.

There are several results in the recent literature that are similar in spirit to the above theorem. In [5], Schacher produces an example of a finite dimensional division algebra $D$ with center an abelian extension of $Q$ with the property that no maximal subfield of $D$ is an abelian extension of $Q$. It can be shown, however, that his example does not arise from a group algebra of a finite group. Given an arbitrary prime $p$ and an arbitrary integer $m \geqq 2$, Ford and Janusz in [3] produce an example of a complex irreducible representation $T$ with character $\chi$ of a finite group $G$ such that $m_{Q}(\chi)=p, \varepsilon_{p^{2}} \notin Q(\chi)$, and for some $r>m, T$ is realizable in $Q(\chi)\left(\varepsilon_{p^{r}}\right)$ but not in any proper subfield. It can be shown, however, that $T$ is also realizable in a subfield $L$ of $Q\left(\varepsilon_{n}\right), n$ the exponent of $G$, where $[L: Q(\chi)]=p$. In [2] an example is found of an irreducible complex representation $T$ with character $\chi$ of a finite group $G$ of order $n$ with the property that $T$ is not realizable in any subfield $L$ of $Q\left(\varepsilon_{n}\right)$ 
with $[L: Q(\chi)]=m_{Q}(\chi)$. It turns out, however, that there exists a prime $q$ and a subfield $L$ of $Q\left(\varepsilon_{n q}\right)$ with $[L: Q(\chi)]=m_{Q}(\chi)$ in which $T$ is realizable. The example in this paper is obtained by suitably modifying the construction we gave in [2]; both the details of the construction and the verification of the properties asserted are much more complicated than in that paper.

Notation and Terminology. We denote the completion of an algebraic number field $K$ at a prime $\pi$ by $K_{\pi}$. If $A$ is a simple component of a group algebra over $Q$, the center of $A$ being $K$, and $\pi_{1}$ and $\pi_{2}$ are primes of $K$ extending the rational prime $p$, then the indices of $A \bigotimes_{K} K_{\pi_{1}}$ and $A \bigotimes_{K} K_{\pi_{2}}$ are equal [6, Corollary 6.3]. We refer to this common value as the $p$-local index of $A$. If $L \supset K$ and $L$ is an abelian extension of $K$, we refer to the local degree (respectively, residue class degree and ramification degree) of a prime $\pi$ of $K$ from $K$ to $L$ as the $p$-local degree (respectively, $p$-residue class degree and $p$-ramification degree) where $\pi$ extends the rational prime $p$. We denote the Galois group of $L$ over $K$ by $\operatorname{Gal}(L / K)$. Let $G$ be a finite group, $T$ a complex irreducible representation of $G$, and $\chi$ the character afforded by $T$. We say that the simple component $A$ of the group algebra of $G$ over $Q(\chi)$ is associated with $\chi$ if the representation of $G$ afforded by a minimal left ideal of $A$ is equivalent to $m_{Q}(\chi) T$. The index of $A$ equals $m_{Q}(\chi)$ and $T$ is realizable in a field $L$ if and only if $L$ splits $A$. In this case, we say that $L$ is a splitting field for $\chi$.

Proof of Theorem 1. Let $p$ be an arbitrary prime and $m$ an arbitrary positive integer. Let $r$ and $s$ be primes with $r \equiv 1+$ $p^{m}\left(\bmod p^{m+1}\right)$ and $s \equiv 1+p^{m-1}\left(\bmod p^{m}\right)$. Let $\gamma \in \operatorname{Gal}\left(Q\left(\varepsilon_{s}\right) / Q\right)$ have order $p^{m-1}$. By the Frobenius density theorem [4, Theorem 5.2], there is a prime $q_{0}$ whose Frobenius automorphism $\left[Q\left(\varepsilon_{s}\right) / Q / q_{0}\right]$ is $\gamma$. Let $q$ be a prime with $q \equiv q_{0}(\bmod s), q \equiv 1+p^{3 m-1}\left(\bmod p^{3 m}\right)$, and $q \equiv 1(\bmod r)$.

Let $F_{0}$ be the subfield of $Q\left(\varepsilon_{s}\right)$ with $\left[Q\left(\varepsilon_{s}\right): F_{0}\right]=p^{m-1}$ and let $F_{1}$ be the subfield of $Q\left(\varepsilon_{r}\right)$ with $\left[Q\left(\varepsilon_{r}\right): F_{1}\right]=p^{m}$. Let $\langle\alpha\rangle=\operatorname{Gal}\left(Q\left(\varepsilon_{r s}\right) /\right.$ $\left.F_{0}\left(\varepsilon_{r}\right)\right)$ and $\langle\beta\rangle=\operatorname{Gal}\left(Q\left(\varepsilon_{r s}\right) / F_{1}\left(\varepsilon_{s}\right)\right)$. Let $K_{0}$ be the fixed field of $Q\left(\varepsilon_{r s}\right)$ under $\langle\alpha \beta\rangle$. Then $K_{0} \cap F_{0}\left(\varepsilon_{r}\right)=K_{0} \cap F_{1}\left(\varepsilon_{s}\right)=F_{0}\left(\varepsilon_{r}\right) \cap F_{1}\left(\varepsilon_{s}\right)=F_{0} F_{1}$ since an element in the first intersection, for example, will be invariant under both $\langle\alpha\rangle$ and $\langle\alpha \beta\rangle$ and so under $\langle\alpha, \beta\rangle$. $\left[K_{0}: F_{0} F_{1}\right]=p^{m-1}$ and $\left[Q\left(\varepsilon_{r s}\right): K_{0}\right]=p^{m}$.

Since $\equiv q q_{0}(\bmod s), q$ splits completely in $F_{0}$. Since $q \equiv 1(\bmod r), q$ also splits completely in $Q\left(\varepsilon_{r}\right)$ and so $q$ splits completely from $Q$ to $F_{0}\left(\varepsilon_{r}\right)$. Because of our choice of $q_{0}, q$ is inertial from $F_{0} F_{1}$ to $F_{1}\left(\varepsilon_{s}\right)$. Thus $q$ is unramified from $F_{0} F_{1}$ to $Q\left(\varepsilon_{r s}\right)$ with residue class degree $p^{m-1}$. Since $K_{0} \cap F_{0}\left(\varepsilon_{r}\right)=F_{0} F_{1}$ and $K_{0}\left(\varepsilon_{r}\right)=Q\left(\varepsilon_{r s}\right)$, we see that $q$ must 
be inertial from $F_{0} F_{1}$ to $K_{0}$. Thus $q$ splits completely from $K_{0}$ to $Q\left(\varepsilon_{r s}\right)$ and has residue class degree $p^{m-1}$ from $Q$ to $K_{0}$.

Let $\zeta$ denote a primitive $q r s p^{2 m}$-th root of unity. Let $E$ be the subfield of $Q\left(\varepsilon_{q}\right)$ with $\left[Q\left(\varepsilon_{q}\right): E\right]=p^{3 m-1}$ and let $\langle\tau\rangle=\operatorname{Gal}\left(Q(\zeta) / E\left(\varepsilon_{r s p 2 m}\right)\right)$. Let $\langle\sigma\rangle=\operatorname{Gal}\left(Q(\zeta) / K_{0}\left(\varepsilon_{q p 2 m}\right)\right)$ and let $K$ be the fixed field of $Q(\zeta)$ under $\langle\boldsymbol{\sigma} \tau\rangle$. As before, $K \cap K_{0}\left(\varepsilon_{q p^{2 m}}\right)=K \cap E\left(_{r s p^{2 m}}\right)=K_{0}\left(\varepsilon_{q p^{2 m}}\right) \cap$ $E\left(\varepsilon_{r s p 2 m}\right)=K_{0} E\left(\varepsilon_{p^{2 m}}\right) .[Q(\zeta): K]=p^{3 m-1}$ and $\left[K: K_{0} E\left(\varepsilon_{p^{2 m}}\right)\right]=p^{m}$.

Since $q$ splits completely from $K_{0}$ to $Q\left(\varepsilon_{r s}\right), q$ splits completely from $K_{0} E\left(\varepsilon_{p^{2 m}}\right)$ to $E\left(_{r s p^{2 m}}\right)$. Since $q$ is totally ramified from $K_{0} E\left(\varepsilon_{p^{2 m}}\right)$ to $K_{0}\left(\varepsilon_{q p^{2 m}}\right)$, we conclude that $q$ must be totally ramified from $K_{0} E\left(\varepsilon_{p^{2 m}}\right)$ to $K$. Since $q \equiv 1\left(\bmod p^{2 m}\right)$ we have determined completely the behavior of $q$ from $Q$ to $K$ and from $K$ to $Q(\zeta)$ : the $q$-ramification degree is $p^{m}$ from $Q$ to $K$ and $p^{2 m-1}$ from $K$ to $Q(\zeta)$ while the $q$-residue class degree is $p^{m-1}$ from $Q$ to $K$ and 1 from $K$ to $Q(\zeta)$. We also note that since $Q(\zeta)=K\left(\varepsilon_{q}\right)$, all primes except $q$ are unramified from $K$ to $Q(\zeta)$. Since $p^{2 m} \geqq 4, K$ is totally imaginary.

Let $G$ by the finite group generated by $w, x, y$, and $z$ subject to the following relations: $w^{p^{5 m-1}}=x^{q}=y^{r}=z^{s}=(x, y)=(x, z)=(y, z)=1$, $w^{p^{3 m-1}}$ central in $G, w^{-1} x w=x^{a}, w^{-1} y w=y^{b}$, and $w^{-1} z w=z^{c}$ where $\tau\left(\varepsilon_{q}\right)=\left(\varepsilon_{q}\right)^{a}, \sigma\left(\varepsilon_{r}\right)=\left(\varepsilon_{r}\right)^{b}$, and $\sigma\left(\varepsilon_{s}\right)=\left(\varepsilon_{s}\right)^{c}$. The cyclic algebra $\mathscr{A}=$ $\left(Q(\zeta), \sigma \tau, \varepsilon_{p^{2 m}}\right)$ is a homomorphic image of the group algebra of $G$ over $Q$ and so there exists a complex irreducible representation $T$ of $G$ with character $\chi$ such that the enveloping algebra of $T$ is $\mathscr{A}$ and $Q(\chi)=K$. The index of equals $m_{Q}(\chi)$.

Since $\mathscr{C}$ is a cyclotomic algebra over a totally imaginary field and only primes over $q$ are ramified from $K$ to $Q(\zeta)$, $\mathscr{A}$ can have nonzero Hasse invariant only at primes of $K$ over $q$ [6, Lemma 4.2]. Since the index of $\mathscr{A}$ is the least common multiple of the indices of $\mathscr{A} \bigotimes_{K} K_{\pi}$ over all primes $\pi$ of $K[1, \mathrm{VII}, \S 5]$, we conclude that $m_{Q}(\chi)$ equals the $q$-local index of $\mathscr{A}$.

The $q$-local index of $\mathscr{A}$ can be computed from [2, Lemma, page 428]. Since $p^{3 m-1}$ is the exact power of $p$ dividing $q-1$ and the $q$-residue class degree from $Q$ to $K$ is $p^{m-1}$, we conclude that $p^{4 m-2}$ is the exact power of $p$ dividing the order of the multiplicative group of the residue class field of $K$ at $q$. Since the $q$-ramification degree from $K$ to $Q(\zeta)$ is $p^{2 m-1}$, we conclude that the $q$-local index of $\mathscr{C}$ is $p$. Thus $m_{Q}(\chi)=p$.

Let $L$ be an abelian extension of $Q$ which is a splitting field for $\mathscr{A}$ and suppose $[L: K]<p^{m}$. If $K \subset L_{0} \subset L$ with $\left[L_{0}: K\right]$ being the full $p$-part of $[L: K]$, then $L_{0}$ must split $\mathscr{A}$ since $\mathscr{A} \bigotimes_{K} L_{0}$ must have index prime to $p$. Thus we may assume that $[L: K]$ is a power of $p$. Since $L$ is abelian over $Q, L \subset Q\left(\varepsilon_{b}\right)$ for some $b$. We clearly may assume that $p$ is the only prime whose square divides $b$. Since $L \supset K, b$ is divisible by $p^{2 m} q r s$ so we may write $b=q r s v$ 
where $(q r s, v)=1$. Let $W$ be the subfield of $K\left(\varepsilon_{v}\right), W \supset K$, such that $[W: K]$ equals the full $p$-part of $\left[K\left(\varepsilon_{v}\right): K\right]$. Since $\varepsilon_{r s} \in K\left(\varepsilon_{q}\right)=Q(\zeta)$, we have $W\left(\varepsilon_{q}\right) \supset L$ and $W \cap K\left(\varepsilon_{q}\right)=K$. Thus $\left[W\left(\varepsilon_{q}\right): W\right]=\left[K\left(\varepsilon_{q}\right): K\right]=$ $p^{3 m-1}$. Since $\operatorname{Gal}\left(W\left(\varepsilon_{q}\right) / W\right)$ is cyclic of order $p^{3 m-1}$, the subfields of $W\left(\varepsilon_{q}\right)$ containing $W$ are linearly ordered and there is one such field for each $p^{i}, 1 \leqq p^{i} \leqq p^{3 m-1}$. Since $\left[K\left(\varepsilon_{r s}\right): K\right]=p^{m},\left[W\left(\varepsilon_{r s}\right): W\right]=p^{m}$. Since we have assumed that $[L: K]<p^{m},[W L: W]<p^{m}$ and so $W L \subset W\left(\varepsilon_{r s}\right)$. Since $(q, v r s)=1, q$ is unramified from $K$ to $L$ and $L \subset W\left(\varepsilon_{r s}\right)$.

Let the prime factorization of $v$ be $p^{i} p_{2} \cdots p_{d}$. Let $W_{1}, \cdots, W_{d}$ be subfields of $W$ such that $K \subset W_{1} \subset K\left(\varepsilon_{p^{i}}\right), K \subset W_{j} \subset K\left(\varepsilon_{p_{j}}\right)$ for $j \geqq 2, L \subset W_{1} W_{2} \cdots W_{d}\left(\varepsilon_{r s}\right)$, but $L$ is not contained in any subfield $V_{1} V_{2} \cdots V_{d}\left(\varepsilon_{r s}\right)$ where $V_{j} \subset W_{j}, j \geqq 1$, and $V_{j}$ is a proper subfield of $W_{j}$ for some $j$. Assume $\left|W_{j}\right| \geqq p^{m}$ for some $j$. Let $V$ be the subfield of $W\left(\varepsilon_{r s}\right)$ generated by the $W_{k}$ with $k \neq j$ and by $\varepsilon_{r s}$. Let $Y=W_{j}$ and let $Y_{0} \subset Y$ with $\left[Y_{0}: K\right]=p^{m-1}$. By the minimality assumption on the $\left\{W_{j}\right\}, L$ is not a subfield of $V Y_{0}$. Since $\operatorname{Gal}(Y V / V)$ is a cyclic $p$-group, the fields intermediate between $Y V$ and $V$ are linearly ordered. Since $Y \cap V=K,\left[Y_{0} V: V\right]=\left[Y_{0}: K\right]=p^{m-1}$. $[L V: V] \leqq[L: K] \leqq p^{m-1} \quad$ so $L V \subset Y_{0} V$. But then $L \subset Y_{0} V$, contradicting our minimality assumption.

We have shown that $\left[W_{j}: K\right] \leqq p^{m-1}$ for $j \geqq 1$. Since $L$ splits $\mathscr{A}$., the $q$-local degree from $K$ to $L$ is divisible by $p$ [1, VII, $\S 5]$. Since $q$ splits completely from $K$ to $K\left(\varepsilon_{r s}\right)$, the $q$-local degree from $K$ to $W_{j}$ must be divisible by $p$ for some $j \geqq 1$. Since $W_{1} \subset K\left(\varepsilon_{p^{i}}\right)$ and $\left[W_{1}: K\right] \leqq p^{m-1}, W_{1} \subset K\left(\varepsilon_{p^{3 m-1}}\right)$. But $q \equiv 1\left(\bmod p^{3 m-1}\right)$ and so the $q$-local degree from $K$ to $W_{1}$ must be one.

We have now shown the existence of a prime $t,(t, p q r s)=1$, such that there is a subfield $S$ of $K\left(\varepsilon_{t}\right), S \supset K$, with $[S: K]=p^{g} \leqq p^{m-1}$ and such that the $q$-local degree from $K$ to $S$ is divisible by $p$. Since $Q\left(\varepsilon_{t}\right) \cap K=Q, S=K S_{0}$ where $S_{0} \subset Q\left(\varepsilon_{t}\right),\left[S_{0}: Q\right] \leqq p^{m-1}$. But the $q$-residue class degree from $Q$ to $K$ is $p^{m-1}$ and so the completion of $S_{0}$ at a prime extending $q$ is contained in the completion of $K$ at a prime extending $q$. This proves that the $q$-local degree from $K$ to $K S_{0}=S$ is 1 and so we conclude that $[L: K] \geqq p^{m}$. Finally, we note that since $q \equiv 1\left(\bmod p^{3 m-1}\right), q \neq 1\left(\bmod p^{3 m}\right), K\left(\varepsilon_{p^{3 m}}\right)$ splits $\mathscr{A}$ and $\left[K\left(\varepsilon_{p^{3} m}\right): K\right]=p^{m}$. This completes the proof of Theorem 1 .

Our final result shows that examples as in Theorem 1 do not exist if we require $Q(\chi)$ to be a cyclotomic field.

THEOREM 2. Let $\chi$ be an irreducible complex character of a finite group $G$ of order $n$ and suppose $Q(\chi)=Q\left(\varepsilon_{r}\right)$ for some $r$. Then there is a splitting field $L$ for $\chi$ with $Q\left(\varepsilon_{n}\right) \supset L \supset Q(\chi)$ and $[L: Q(\chi)]=m_{Q}(\chi)$. 
Proof. This result was proved in [2] provided $m_{Q}(\chi) \geqq 3$. If $r \geqq 3$, the argument of that paper is still valid even if $m_{Q}(\chi)=2$. Thus we may assume that $m_{Q}(\chi)=2$ and $Q(\chi)=Q$. Let $\mathscr{A}$ be the simple component of $Q G$ which is associated with $\chi$. If $8 \mid n$, set $t=-2 p_{1} \cdots p_{u}$, where $p_{1}, \cdots, p_{u}$ are the distinct odd primes dividing n. Then $Q(\sqrt{t}) \subset Q\left(\varepsilon_{n}\right)$ and the $p$-local degree from $Q$ to $Q(\sqrt{t})$ is 2 for all primes $p$ of $Q$ at which $\mathscr{A}$ could have nonzero Hasse invariant. It follows that $Q(\sqrt{t})$ splits $\mathscr{A}$ and so we may assume that $8 \nmid n$. By [6, Theorem 9.1], 4|n. Let $Q\left(\varepsilon_{n}\right) \supset K \supset Q$ be such that $[K: Q]$ is odd and $\left[Q\left(\varepsilon_{n}\right): K\right]$ is a power of 2 . By the Brauer-Witt theorem [6, page 31], there is a hyperelementary subgroup $F$ of $G$, a normal subgroup $N$ of $F$, and a linear character $\psi$ of $N$ such that

$\otimes_{Q} K$ is similar to a cyclotomic algebra $(K(\psi) / K, \beta)$ where the values of $\beta$ are values of $\psi$ on $N$ and where $\operatorname{Gal}(K(\psi) / K) \cong F / N$. If $|N|$ is odd, then $(K(\psi) / K, \beta)^{|N|} \sim(K(\psi) / K, 1)$ is split, contradicting $m_{Q}(\chi)=2$. Thus 2||$N \mid$ and so $|F| N \mid=2$. It follows that the quadratic subfield of $Q(\psi)$ is our desired splitting field for $\chi$. This completes the proof of Theorem 2 .

It would be interesting to replace the $n$ in the statement of Theorem 2 by the exponent of $G$. If $Q(\chi) \neq Q$, this result is already in [2]. If 8 divides the exponent of $G$, the argument of Theorem 2 applies. There is only difficulty if $Q(\chi)=Q, m_{Q}(\chi)=2$, and, in the notation of Theorem 2 , $\&$ has nonzero invariants at $2, \infty$, and some other primes of $Q$. The problem, of course, is that the natural candidate for a splitting field, $Q(\sqrt{v})$ with $v=-p_{1} \cdots p_{u}$, need not split $\mathscr{C}$ at the prime 2 . We have not been able to resolve this difficulty.

\section{REFERENCES}

1. M. Deuring, Algebren, Springer, Berlin, 1935.

2. B. Fein, Minimal splitting fields for group representations, Pacific J. Math. 51 (1974), 427-431.

3. C. Ford and G. Janusz, Examples in the theory of the Schur group, Bull. Amer. Math. Soc., 79 (1973), 1233-1235.

4. G. Janusz, Algebraic Number Fields, Academic Press, New York, 1973.

5. M. Schacher, Cyclotomic splitting fields, Proc. Amer. Math. Soc., 25 (1970), 630-633.

6. T. Yamada, The Schur subgroup of the Brauer group, Lecture Notes in Math., Vol. 397, Springer, Berlin-Heidelberg-New York, 1974.

Received September 23, 1975. This work was supported in part by NSF Grant GP29068. 



\title{
PACIFIC JOURNAL OF MATHEMATICS
}

\section{EDITORS}

RICHARD ARENS (Managing Editor)

University of California

Los Angeles, CA 90024

Charles W. Curtis

University of Oregon

Eugene, OR 97403

C. C. MOORE

University of California

Berkeley, CA 94720
J. DUGUNDJI

Department of Mathematics

University of Southern California

Los Angeles, CA 90007

R. Finn and J. Milgram

Stanford University

Stanford, CA 94305

\section{ASSOCIATE EDITORS}
E. F. BECKENBACH
B. H. Neumann
F. WOLF
K. YosHidA

\section{SUPPORTING INSTITUTIONS}

\author{
UNIVERSITY OF BRITISH COLUMBIA \\ CALIFORNIA INSTITUTE OF TECHNOLOGY \\ UNIVERSITY OF CALIFORNIA \\ MONTANA STATE UNIVERSITY \\ UNIVERSITY OF NEVADA, RENO \\ NEW MEXICO STATE UNIVERSITY \\ OREGON STATE UNIVERSITY \\ UNIVERSITY OF OREGON
}

\author{
UNIVERSITY OF SOUTHERN CALIFORNIA \\ STANFORD UNIVERSITY \\ UNIVERSITY OF HAWAII \\ UNIVERSITY OF TOKYO \\ UNIVERSITY OF UTAH \\ WASHINGTON STATE UNIVERSITY \\ UNIVERSITY OF WASHINGTON
}

The Supporting Institutions listed above contribute to the cost of publication of this Journal, but they are not owners or publishers and have no responsibility for its content or policies.

Mathematical papers intended for publication in the Pacific Journal of Mathematics should be in typed form or offset-reproduced, (not dittoed), double spaced with large margins. Please do not use built up fractions in the text of the manuscript. However, you may use them in the displayed equations. Underline Greek letters in red, German in green, and script in blue. The first paragraph or two must be capable of being used separately as a synopsis of the entire paper. Items of the bibliography should not be cited there unless absolutely necessary, in which case they must be identified by author and journal, rather than by item number. Manuscripts, in triplicate, may be sent to any one of the editors. Please classify according to the scheme of Math. Reviews, Index to Vol. 39. All other communications should be addressed to the managing editor, or Elaine Barth, University of California, Los Angeles, California, 90024.

50 reprints to each author are provided free for each article, only if page charges have been substantially paid. Additional copies may be obtained at cost in multiples of 50 .

The Pacific Journal of Mathematics is issued monthly as of January 1966. Regular subscription rate: $\$ 72.00$ a year (6 Vols., 12 issues). Special rate: $\$ 36.00$ a year to individual members of supporting institutions.

Subscriptions, orders for numbers issued in the last three calendar years, and changes of address should be sent to Pacific Journal of Mathematics, P.O. Box 969, Carmel Valley, CA 93924, U.S.A. Older back numbers obtainable from Kraus Periodicals Co., Route 100, Millwood, NY 10546.

PUBLISHED BY PACIFIC JOURNAL OF MATHEMATICS, A NON-PROFIT CORPORATION

Printed at Kokusai Bunken Insatsusha (International Academic Printing Co., Ltd.). 8-8, 3-chome, Takadanobaba, Shinjuku-ku, Tokyo 160, Japan.

Copyright (C) 1978 by Pacific Journal of Mathematics Manufactured and first issued in Japan 


\section{Pacific Journal of Mathematics}

Vol. 77, No. $2 \quad$ February, 1978

Graham Donald Allen, Duals of Lorentz spaces ................... 287

Gert Einar Torsten Almkvist, The number of nonfree components in the decomposition of symmetric powers in characteristic p .......... 293

John J. Buoni and Bhushan L. Wadhwa, On joint numerical ranges ...... 303

Joseph Eugene Collison, Central moments for arithmetic functions . . . . . . 307

Michael Walter Davis, Smooth G-manifolds as collections of fiber

bundles ........................................ 315

Michael E. Detlefsen, Symmetric sublattices of a Noether lattice......... 365

David Downing, Surjectivity results for $\phi$-accretive set-valued

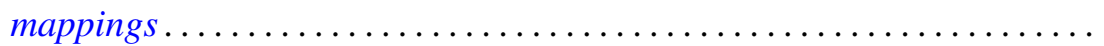

David Allyn Drake and Dieter Jungnickel, Klingenberg structures and partial designs. II. Regularity and uniformity ................. 389

Edward George Effros and Jonathan Rosenberg, $C^{*}$-algebras with approximately inner flip .......................... 417

Burton I. Fein, Minimal splitting fields for group representations. II. . . . . 445

Benjamin Rigler Halpern, A general coincidence theory ............. 451

Masamitsu Mori, A vanishing theorem for the mod $p$ Massey-Peterson spectral sequence ................................ 473

John C. Oxtoby and Vidhu S. Prasad, Homeomorphic measures in the

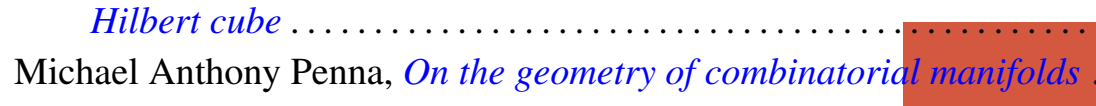

Robert Ralph Phelps, Gaussian null sets and differentiability of Lipschitz map on Banach spaces........................

Herbert Silverman, Evelyn Marie Silvia and D. N. Telage, Locally univalent functions and coefficient distortions...

Donald Curtis Taylor, The strong bidual of $\Gamma(K)$

Willie Taylor, On the oscillatory and asymptotic behavior of solutions of fifth order selfadjoint differential equations ...........

Fu-Chien Tzung, Sufficient conditions for the set of Hausdorff compactifications to be a lattice. 